Louisiana State University

LSU Digital Commons

Faculty Publications

Department of Mathematics

$1-1-2015$

\title{
Continuous-discrete observers for time-varying nonlinear systems: A tutorial on recent results
}

\author{
Frederic Mazenc \\ Laboratoire des Signaux et Systèmes \\ Vincent Andrieu \\ Université de Lyon \\ Michael Malisoff \\ Louisiana State University
}

Follow this and additional works at: https://digitalcommons.Isu.edu/mathematics_pubs

\section{Recommended Citation}

Mazenc, F., Andrieu, V., \& Malisoff, M. (2015). Continuous-discrete observers for time-varying nonlinear systems: A tutorial on recent results. SIAM Conference on Control and Its Applications 2015, 181-188. https://doi.org/10.1137/1.9781611974072.26

This Conference Proceeding is brought to you for free and open access by the Department of Mathematics at LSU Digital Commons. It has been accepted for inclusion in Faculty Publications by an authorized administrator of LSU Digital Commons. For more information, please contact ir@lsu.edu. 
archives-ouvertes

\title{
Continuous-Discrete Observers for Time-Varying Nonlinear Systems: A Tutorial on Recent Results
}

\author{
Frederic Mazenc, Vincent Andrieu, Michael Malisoff
}

\section{To cite this version:}

Frederic Mazenc, Vincent Andrieu, Michael Malisoff. Continuous-Discrete Observers for Time-Varying Nonlinear Systems: A Tutorial on Recent Results. 2015 SIAM Conference on Control and its Applications, Jul 2015, Paris, France. 10.1137/1.9781611974072.26 . hal-01257347

\section{HAL Id: hal-01257347 \\ https://hal.inria.fr/hal-01257347}

Submitted on 16 Jan 2016

HAL is a multi-disciplinary open access archive for the deposit and dissemination of scientific research documents, whether they are published or not. The documents may come from teaching and research institutions in France or abroad, or from public or private research centers.
L'archive ouverte pluridisciplinaire HAL, est destinée au dépôt et à la diffusion de documents scientifiques de niveau recherche, publiés ou non, émanant des établissements d'enseignement et de recherche français ou étrangers, des laboratoires publics ou privés. 


\title{
Continuous-Discrete Observers for Time-Varying Nonlinear Systems: A Tutorial on Recent Results *
}

\author{
Frederic Mazenc $^{\dagger} \quad$ Vincent Andrieu ${ }^{\ddagger} \quad$ Michael Malisoff $§$
}

\begin{abstract}
Continuous-discrete systems can occur when the plant state evolves in continuous time but the output values are only available at discrete instants. Continuous-discrete observers have the valuable property that the observation error between the true state of the system and the observer state converges to zero in a uniform way. The design of continuousdiscrete observers can often be done by building framers, which provide componentwise upper and lower bounds for the plant state. This paper is a tutorial on these approaches, highlighting recent results in the literature, and also providing previously unpublished, original results which are not being simultaneously submitted elsewhere.
\end{abstract}

\section{Introduction}

The search for more effective designs for observers for nonlinear systems has led to a substantial and complex literature $[3,4,5,9,15,20,21]$. The work is motivated by a plethora of real world applications where it may be difficult or impossible to measure the state variables. Then the goal is to use output measurements to design an observer for the state such that the observation error between the observer and the state converges to 0 as time goes to infinity. The well known literature on observers is largely for systems with continuous measurements; see, e.g., [21] for results based on writing the differential equation satisfied by the estimation error as a linear parameter varying system.

However, in real world applications, the output is

*Supported by ANR LIMICOS Contract 12 BS03 00501 (Andrieu) and NSF Grant 1102348 (Malisoff).

${ }^{\dagger}$ Mazenc is with EPI DISCO INRIA-Saclay, the Laboratoire des Signaux et Systèmes (L2S, UMR CNRS 8506), CNRS, CentraleSupélec, Université Paris-Sud, 3 rue Joliot Curie, 91192, Gifsur-Yvette, France (frederic.mazenc@12s.centralesupelec.fr).

$¥$ Andrieu is with AGEP, Université de Lyon, Domaine Universitaire de la Doua, CPE, Batiment G, 2ème étage, 43 bd du 11 Novembre 1918, 69622 Villeurbanne Cedex, France and Bergische Universität Wuppertal, Fachbereich C - Mathematik und Naturwissenschaften, Arbeitsgruppe Funktionalanalysis, Gaußstraße 20, 42097, Wuppertal, Germany (vincent.andrieu@gmail.com).

$\S$ Malisoff is with Department of Mathematics, Louisiana State University, Baton Rouge, LA 70803-4918, USA (malisoff@lsu.edu). often unavailable for continuous measurement. Since the dynamics are in continuous time and the output is only available for measurement at discrete instants, this produces a continuous-discrete system. There is now a large literature on observer designs for continuousdiscrete systems that spans over forty years. See, e.g., the work [14] of Jazwinski, which used a continuousdiscrete Kalman filter to solve a filtering problem for stochastic continuous-discrete time systems.

The high gain observer approach in [11] was extended to continuous-discrete systems in [8], where the impulsive correction gain is found using a continuousdiscrete Riccati equation. The robustness of observers under discretization was studied in [5], and $[1,10,15]$ used output predictors to design observers; see also the works $[4,8,13,17,19]$. The paper [2] designed continuous-discrete observers for nonlinear continuous time systems, where the input of the system satisfies a persistent excitation condition, and [18] covers systems that are linear in the state and have known inputs. For an interesting application, see [6] for continuous-discrete observers for emulsion polymerization reactors.

In the recent work [16], we built on [4], by finding a new class of continuous-discrete observers for continuous time Lipschitz systems with discrete measurements. As in [4] and [8], the continuous-discrete observers in [16] are obtained in two steps. First, when the output is not available for measurement, the state estimate is computed by integrating the model. Then, when a measurement occurs, the observer makes an impulsive correction to the estimated state.

The works [4] and [9] used this two step approach to show that when no measurement occurs, the estimation error is a solution of an appropriate unknown linear parameter varying system. This led to a construction of a framer, meaning, an upper and lower bound for the solution vector, in a vector sense, which made it possible to design correction terms that ensure that the estimation error asymptotically converges to zero. However, [4] and [9] find the framer by integrating a system with commutation, which does not lead to an explicit analytic expression for the framer, and [17] is limited to linear systems. By contrast, [16] used an 
approach from [7] on cooperative systems [12] to get analytic constructions of framers, which can be useful for applications where explicit expressions are needed.

This paper provides a tutorial that explains some of the preceding advances precisely, including the motivation for the assumptions and methods and the value added by our recent contributions $[4,9,16]$, while also stating and proving some previously unpublished, original results on framers that are not being simultaneously submitted elsewhere. We believe that our tutorial will fill an important void in the literature, and increase the control community's appreciation for, and understanding of, continuous-discrete observers. In the next section, we provide the relevant definitions. In Section 3, we discuss the work [21] of Zemouche and others in the continuous time case, which illustrates one of the recurring themes in this article, namely, the possibility of using linear matrix inequalities (or LMIs), and therefore also LMI solvers, to design asymptotic observers for nonlinear systems. Then in Section 4, we discuss an extension of [21] to the discrete time measurement case, based on computing a reachable set for controlled systems and solving LMIs.

In Section 5, we discuss our alternative approach to continuous-discrete observers, which is based on designing framers, including results that have not appeared before. In Section 6, we use our framers to formulate our latest theorem on observers. In Section 7, we show how our closed form expressions for the framers allow us to check the assumptions of our theorem using linear matrix inequalities. In Section 8, we summarize our work and suggest future research topics. For novel applications of some of the theory in this paper to pendulum and robotic DC motor dynamics, see [16].

\section{Notation, Definitions, and Basic Result}

Throughout the sequel, we omit arguments of functions, when they are clear from the context, and the dimensions of the matrices are arbitrary. We set $\mathbb{N}=$ $\{1,2, \ldots\}$. The $k \times n$ matrix all of whose entries are 0 will also be denoted by 0 , and we use $A=\left[a_{i j}\right]$ to indicate that an arbitrary matrix $A \in \mathbb{R}^{k \times n}$ has $a_{i j}$ in its $i$ th row and $j$ th column for each $i \in\{1,2, \ldots, k\}$ and $j \in\{1,2, \ldots, n\}$. Also, $I_{s}$ is the identity matrix in any dimension $s$. The usual Euclidean norm $\sqrt{x_{1}^{2}+\ldots+x_{n}^{2}}$ of vectors and the induced norm of matrices are denoted by $|\cdot|$. All inequalities and maxima are componentwise, i.e., if $A=\left[a_{i j}\right]$ and $B=\left[b_{i j}\right]$ are matrices of the same dimensions, then we use $A \leq B$ to mean that $a_{i j} \leq b_{i j}$ for all $i$ and $j$, and $\max \{A, B\}$ is the matrix $C=\left[c_{i j}\right]$ where $c_{i j}=\max \left\{a_{i j}, b_{i j}\right\}$ for all $i$ and $j$. A square matrix is cooperative or Metzler provided all of its off-diagonal entries are non- negative. We use $T$ to denote transpose. For each $r \in \mathbb{N}$ and each function $\mathcal{F}:[0, \infty) \rightarrow \mathbb{R}^{r}$, we use the left limits $\mathcal{F}\left(t_{-}\right)=\lim _{s \rightarrow t, s<t} \mathcal{F}(s)$. A function $\varphi_{*}: \mathbb{R} \times \mathbb{R}^{n} \rightarrow \mathbb{R}^{n}$ is uniformly Lipschitz in its second argument provided there is a constant $L>0$ such that $\left|\varphi_{*}(t, x)-\varphi_{*}(t, y)\right| \leq L|x-y|$ holds for all $t \in \mathbb{R}$, $x \in \mathbb{R}^{n}$, and $y \in \mathbb{R}^{n}$. For any square matrices $A$ and $B$ in $\mathbb{R}^{n \times n}$, we use $A \preccurlyeq B$ (resp., $A \succ B$ ) to mean that $X^{\top}(A-B) X \leq 0$ for all $x \in \mathbb{R}^{n}$ (resp., $X^{\top}(A-B) X>0$ for all $\left.X \in \mathbb{R}^{n} \backslash\{0\}\right)$. We use Conv to denote the closed convex hull.

\section{Background on Continuous Time Observers}

Consider the continuous-discrete system

$$
\dot{x}(t)=A x(t)+\phi(x(t), u(t))
$$

with discrete output measurements at the known sample times $\left\{t_{k}\right\}_{k=1}^{\infty}$ given by

$$
y_{k}=C x\left(t_{k}\right), t_{k+1}=t_{k}+\delta_{k}
$$

where $A$ and $C$ are constant matrices, and the $\delta_{k}$ 's represent the sampling delays. The function $u(t)$ can present an open or closed loop control, and is assumed to be continuous. We make this assumption on (3.1):

Assumption 1. The pair $(A, C) \in \mathbb{R}^{n \times n} \times \mathbb{R}^{p \times n}$ is observable. Also, for each pair $(i, j)$ of values in $\{1,2, \ldots, n\}$, there is a positive real number $b_{i j}$ such that

$$
\left|\frac{\partial \phi_{i}}{\partial x_{j}}(x, u)\right| \leq b_{i j}
$$

for all $(x, u) \in \mathbb{R}^{n} \times \mathbb{R}^{p}$.

By a continuous-discrete observer for (3.1), we mean a system of the form

$$
\left\{\begin{array}{l}
\dot{\hat{x}}(t)=A \hat{x}(t)+\phi(\hat{x}(t), u(t)), t \in\left[t_{k}, t_{k+1}\right) \\
\hat{x}\left(t_{k}\right)=\hat{x}\left(t_{k}^{-}\right)+K\left(y_{k}-C \hat{x}\left(t_{k}^{-}\right)\right)
\end{array}\right.
$$

for all $k \geq 0$, where

$$
\hat{x}\left(t_{k}^{-}\right)=\lim _{t \rightarrow t_{k}, t<t_{k}} \hat{x}(t) .
$$

Then the estimation problem is that of selecting $K$ to ensure that

$$
\lim _{t \rightarrow+\infty}|x(t)-\hat{x}(t)|=0
$$

for all initial conditions.

To motivate our search for $K$, we recall the results from [21] on the continuous time case where $y(t)=$ $C x(t)$. Let $\mathcal{R}$ be the set of all matrices in $\mathbb{R}^{n \times n}$ such that for each matrix $R=\left[r_{i j}\right]$ in $\mathcal{R}$ and each pair $(i, j)$, the corresponding entry $r_{i j}$ is either $b_{i j}$ or $-b_{i j}$. We then have the following result from [21]: 
THEOREM 3.1. If there exist a positive definite symmetric matrix $P \in \mathbb{R}^{n \times n}$ and a matrix $L \in \mathbb{R}^{p \times n}$ such that the $L M I$

$$
(A+R)^{\top} P+P(A+R)-C^{\top} L-L^{\top} C<0
$$

holds for all $R \in \mathcal{R}$, then the system

$$
\begin{aligned}
\dot{\hat{x}}(t)= & A \hat{x}(t)+\phi(\hat{x}(t), u(t)) \\
& +P^{-1} L^{\top}(y(t)-C \hat{x}(t))
\end{aligned}
$$

is an asymptotic observer for (3.1) in the continuous time case where $y(t)=C x(t)$, i.e., the limit (3.6) holds for all initial conditions for (3.1) and (3.8).

Although Theorem 3.1 does not cover discrete output observations, it has the important feature that the sufficient condition is stated in terms of LMIs, which makes it possible to check the assumptions using LMI solvers [20]. This contrasts with traditional LMI approaches, which are usually limited to linear time invariant systems. We next explain our approach from [4] for extending the basic properties of the observer (3.8) to systems with discrete output measurements.

\section{Discrete Output Measurements}

To motivate our extension of [21] to the case of discrete output observations, let $\delta>0$ be any constant, and consider the error system

$$
\left\{\begin{array}{c}
\dot{e}(t)=A e(t)+\Delta \phi(\hat{x}(t), u(t), e(t)) \text { for all } \\
t \in[k \delta,(k+1) \delta) \text { and } k \geq 0 \\
e(k \delta)=\left(I_{n}-K C\right) e\left(k \delta^{-}\right) \text {for all } k \geq 0 .
\end{array}\right.
$$

where $\Delta \phi(\hat{x}, u, e)=\phi(\hat{x}, u)-\phi(\hat{x}-e, u)$ and $e\left(k \delta^{-}\right)=$ $\lim _{t \rightarrow k \delta^{-}} e(t)$. Assuming that $\phi$ is $C^{1}$ in its first argument (i.e., the state) and that there are constants $b_{i j}>0$ such that $(3.3)$ holds for all $(x, u) \in \mathbb{R}^{n} \times \mathbb{R}^{p}$ and all pairs $(i, j)$, the Mean Value Theorem gives $\Delta \phi_{i}(\hat{x}, u, e)=\left(\partial \phi_{i} / \partial x\right)\left(z_{i}(\hat{x}, e, u), u\right) e$ for all $i$ for suitable points $z_{i}(\hat{x}, e, u)$. Hence, between any two measurements, the error is a solution of $\dot{e}(t)=A e(t)+$ $V(t) e(t)$, where $V(t)=\left[v_{i j}(t)\right] \in \mathbb{R}^{n \times n}$ is a matrix each of whose entries is bounded by $b_{i j}$.

For each choice of $e_{0} \in \mathbb{R}^{n}$ and each real number $\delta>0$, let $\mathcal{A}_{\delta}(e) \subset \mathbb{R}^{n}$ denote the reachability set at time $\delta$ with the control constraint that

$$
\left|v_{i j}(t)\right| \leq b_{i j}
$$

for all $i$ and $j$ and $t \geq 0$. This means that for each $e_{1}$ in $\mathcal{A}_{\delta}\left(e_{0}\right)$, there exists a function $V(t)=\left[v_{i j}(t)\right]$ such that (4.9) holds for all $t$ in $[0, \delta]$ and all pairs $(i, j)$, and such that the solution $e(t)$ of $\dot{e}(t)=A e(t)+V(t) e(t)$ starting from $e_{0}$ satisfies $e(\delta)=e_{1}$. We then have the following discrete time extension from [4]:
THEOREM 4.1. Assume that there are constants $b_{i j}>0$ such that (3.3) holds for all $(x, u) \in \mathbb{R}^{n} \times \mathbb{R}^{p}$ and all pairs $(i, j)$ and that $\phi$ is $C^{1}$. If there exist a finite set of matrix valued functions $\mathcal{S}=\left\{M_{1}, M_{2}, \ldots, M_{\ell}\right\}$ mapping $[0, \infty)$ into $\mathbb{R}^{n \times n}$, a symmetric positive definite matrix $P$ in $\mathbb{R}^{n \times n}$, and $a W \in \mathbb{R}^{n \times p}$ such that

$$
\mathcal{A}_{\delta}(e) \subseteq \operatorname{Conv}\left\{M_{i}(\delta) e: 1 \leq i \leq \ell\right\}
$$

holds for all $e \in \mathbb{R}^{n}$, and such that the matrix inequality

$$
\left[\begin{array}{ll}
P & M_{i}^{\top}(\delta)\left(P-C^{\top} W^{\top}\right) \\
(P-W C) M_{i}(\delta) & P
\end{array}\right]
$$

is positive definite for all $i \in\{1,2, \ldots, \ell\}$, then the choice

$$
K=P^{-1} W
$$

in our observer (3.8) achieves our estimation goal (3.6).

The preceding theorem can be summarized as follows. The first step is the computation of a reachable set for a controlled system. Exact computation of this set is not needed, but only the upper approximation (4.10) in terms of the $M_{i}$ 's for $1 \leq i \leq \ell$. For lower triangular systems, such an upper approximation was given in [4]. The second step is to check the positive definiteness of the matrices (4.11). For applications of Theorem 4.1, including cases where the system is uniformly observable, see [4]. A possible drawback of the preceding approach is that it does not lead to analytic formulas for framers. We next provide an alternative approach to continuousdiscrete observers that has the advantage of providing closed form expressions for framers.

\section{Background on Framers}

In this section, we present several results on framers for time-varying linear systems that we use in the next section to build our continuous-discrete observers for nonlinear systems. Take any linear time-varying system

$$
\dot{x}(t)=\mathcal{M}(t) x(t)
$$

with state space $\mathbb{R}^{n}$, where all entries of $\mathcal{M}:[0, \infty) \rightarrow$ $\mathbb{R}^{n \times n}$ are continuous. Let $\varrho\left(t, t_{0}\right)$ denote the fundamental solution of the system (5.13), meaning, $(\partial \varrho / \partial t)\left(t, t_{0}\right)=\mathcal{M}(t) \varrho\left(t, t_{0}\right)$ and $\varrho\left(t_{0}, t_{0}\right)=I_{n}$ hold for all $t_{0} \geq 0$ and $t \geq t_{0}$. In this section, we derive lower and upper bounds for the function $\Gamma(t)=\varrho(t, 0)$. Note for later use that the solution $\phi$ of

$$
\frac{\partial \phi}{\partial t}\left(t, x_{0}\right)=\mathcal{M}(t) \phi\left(t, x_{0}\right), \quad \phi\left(0, x_{0}\right)=x_{0}
$$

satisfies $\phi\left(t, x_{0}\right)=\Gamma(t) x_{0}$ for all $t \geq 0$ and $x_{0} \in \mathbb{R}^{n}$. Our next lemma on framers is a key ingredient needed to prove our main result on framers. It assumes: 
Assumption 2. There are two constant Metzler matrices $\overline{\mathcal{M}} \in \mathbb{R}^{n \times n}$ and $\underline{\mathcal{M}} \in \mathbb{R}^{n \times n}$ such that

$$
\underline{\mathcal{M}} \leq \mathcal{M}(t) \leq \overline{\mathcal{M}} \text { for all } t \geq 0 \text {. }
$$

Also, $\mathcal{M}: \mathbb{R} \rightarrow \mathbb{R}^{n \times n}$ is continuous.

The following is shown in [16]:

LEMMA 5.1. If Assumption 2 holds, then $\exp (\underline{\mathcal{M}} t) \leq$ $\Gamma(t) \leq \exp (\overline{\mathcal{M}} t)$ hold for all $t \geq 0$.

Next, we consider the system (5.13) under the following much weaker assumption than Assumption 2:

Assumption 3. The matrix valued function $\mathcal{M}$ : $[0, \infty) \rightarrow \mathbb{R}^{n \times n}$ is bounded and continuous.

Assumption 3 allows us to pick functions $\mathcal{K}: \mathbb{R} \rightarrow$ $\mathbb{R}^{n \times n}$ and $\mathcal{L}: \mathbb{R} \rightarrow \mathbb{R}^{n \times n}$, a constant matrix $\overline{\mathcal{L}} \geq 0$, and constant Metzler matrices $\overline{\mathcal{K}}$ and $\underline{\mathcal{K}}$ such that

$$
\begin{aligned}
& \mathcal{M}(t)=\mathcal{K}(t)-\mathcal{L}(t), \quad 0 \leq \mathcal{L}(t) \leq \overline{\mathcal{L}} \\
& \text { and } \underline{\mathcal{K}} \leq \mathcal{K}(t) \leq \overline{\mathcal{K}}
\end{aligned}
$$

hold for all $t \geq 0$. Since $\mathcal{M}$ is bounded, the decomposition (5.16) can be obtained by replacing each entry of $\mathcal{M}(t)=\left[m_{i j}(t)\right]$ by $m_{i j}(t)+\bar{B}$ for a big enough constant $\bar{B}>0$ to produce the Metzler matrices $\mathcal{K}(t)$ for each $t$, and then letting $\mathcal{L}=\overline{\mathcal{L}}$ be the constant matrix with $\bar{B}$ as each entry, but other decompositions of the type (5.16) exist. In [16], the following is shown:

LEMMA 5.2. Let the system (5.13) satisfy Assumption 3 , and let $\mathcal{L}, \mathcal{K}, \overline{\mathcal{L}} \in \mathbb{R}^{n \times n}, \overline{\mathcal{K}} \in \mathbb{R}^{n \times n}$, and $\underline{\mathcal{K}} \in$ $\mathbb{R}^{n \times n}$ satisfy the preceding requirements. Define the $C^{1}$ functions $\underline{\Gamma}:[0, \infty) \rightarrow \mathbb{R}^{n \times n}$ and $\bar{\Gamma}:[0, \infty) \rightarrow \mathbb{R}^{n \times n}$ by

$$
\begin{aligned}
& \underline{\Gamma}(t)=e^{\underline{\mathcal{K}} t}+\frac{1}{2}\left[e^{(\overline{\mathcal{K}}-\overline{\mathcal{L}}) t}-e^{(\overline{\mathcal{K}}+\overline{\mathcal{L}}) t}\right] \text { and } \\
& \bar{\Gamma}(t)=\frac{1}{2}\left[e^{(\overline{\mathcal{K}}+\overline{\mathcal{L}}) t}+e^{(\overline{\mathcal{K}}-\overline{\mathcal{L}}) t}\right] .
\end{aligned}
$$

Then $\underline{\Gamma}(t) \leq \Gamma(t) \leq \bar{\Gamma}(t)$ hold for all $t \geq 0$.

The proof of Lemma 5.2 is based on applying Lemma 5.1 to the $2 n$ dimensional system

$$
\dot{\Lambda}(t)=\mathcal{H}(t) \Lambda(t)
$$

where

$$
\mathcal{H}(t)=\left[\begin{array}{ll}
\mathcal{K}(t) & \mathcal{L}(t) \\
\mathcal{L}(t) & \mathcal{K}(t)
\end{array}\right]
$$

Two key novel features of Lemma 5.1 are that it produces many framers, for different $\mathcal{K}$ 's and $\mathcal{L}$ 's, and that one only needs $\overline{\mathcal{K}}, \overline{\mathcal{L}}$, and $\underline{\mathcal{K}}$ to build the framers.
However, it is useful to note that one can build framers by applying Lemma 5.1 to lower dimensional systems, where the embedding of the $n$ dimensional system is into a larger system of dimension strictly less than $2 n$. We next present new original results in this direction that have not been submitted elsewhere. For each continuous matrix $D(t)$ of size $n \times n$ having the fundamental matrix $q\left(t, t_{0}\right)$, we set $\phi_{D}(t)=q(t, 0)$. Then $\phi_{D}(0)=I_{n}$ and $\phi_{M}(0)=\varrho(t, 0)=\Gamma(t)$. We also set $A^{+}=\max \{0, A\}$ and $A^{-}=A^{+}-A$ for any matrix $A$, so $A^{+} \geq 0$ and $A^{-} \geq 0$. We prove:

Proposition 5.1. Let Assumption 2 hold. Assume that there is a constant matrix $P \in \mathbb{R}^{n \times n}$ such that

$$
P \mathcal{M}(t) P^{-1}=B(t)=\left[\begin{array}{cc}
B_{1}(t) & B_{2}(t) \\
0 & B_{3}(t)
\end{array}\right]
$$

where $B_{1}$ is valued in $\mathbb{R}^{s \times s}$ for some $s \in(0, n)$ and $B_{2}$ and $B_{3}$ are of suitable dimensions. Set $K=\left[I_{s}, 0\right] \in$ $\mathbb{R}^{s \times n}, R=P^{-1}, G=R^{+} K^{\top}$, and $F=R^{-} K^{\top}$, and set

$$
\begin{aligned}
& \mathcal{L}_{a}(t)=P^{+} e^{\underline{\mathcal{M}} t}-P^{-} e^{\overline{\mathcal{M}} t} \text { and } \\
& \mathcal{L}_{b}(t)=P^{+} e^{\overline{\mathcal{M}} t}-P^{-} e \underline{\underline{\mathcal{M}}} t
\end{aligned}
$$

Then

$$
\begin{aligned}
K\left(\mathcal{L}_{a}(t) G-\mathcal{L}_{b}(t) F\right) & \leq \phi_{B_{1}}(t) \\
& \leq K\left(\mathcal{L}_{b}(t) G-\mathcal{L}_{a}(t) F\right)
\end{aligned}
$$

hold for all $t \geq 0$.

Proof: Throughout the proof, all equalities and inequalities hold for all $t \geq 0$. Our condition (5.20) implies that $P\left(\partial \phi_{\mathcal{M}} / \partial t\right)(t) P^{-1}=P \mathcal{M}(t) P^{-1} P \phi_{\mathcal{M}}(t) P^{-1}=$ $B(t) P \phi_{\mathcal{M}}(t) P^{-1}$. Hence, by the uniqueness of solutions property, $\phi_{B}(t)=P \phi_{\mathcal{M}}(t) P^{-1}$, so Assumption 2 gives

$$
e^{\underline{\mathcal{M}} t} \leq R \phi_{B}(t) P=\phi_{\mathcal{M}}(t) \leq e^{\overline{\mathcal{M}} t} .
$$

Consequently, since $P^{+} \geq 0$ and $P^{-} \geq 0$, we have

$$
\begin{gathered}
P^{+} e \underline{\underline{\mathcal{M}}} t \leq P^{+} R \phi_{B}(t) P \leq P^{+} e^{\overline{\mathcal{M}} t} \text { and } \\
P^{-} e^{\underline{\mathcal{M}} t} \leq P^{-} R \phi_{B}(t) P \leq P^{-} e^{\overline{\mathcal{M}} t} .
\end{gathered}
$$

Since $R=P^{-1}$, it follows that

$$
\begin{aligned}
& P^{+} e \underline{\mathcal{M}} t-P^{-} e^{\overline{\mathcal{M}} t} \leq \phi_{B}(t) P \\
& \leq P^{+} e^{\overline{\mathcal{M}} t}-P^{-} e \underline{\underline{\mathcal{M}} t}
\end{aligned}
$$

and therefore also

$$
\begin{aligned}
& {\left[P^{+} e^{\underline{\mathcal{M}} t}-P^{-} e^{\overline{\mathcal{M}} t}\right] R^{+} \leq \phi_{B}(t) P R^{+}} \\
& \leq\left[P^{+} e^{\overline{\mathcal{M}} t}-P^{-} e^{\underline{\mathcal{M}}} t\right] R^{+} \text {and } \\
& {\left[P^{+} e^{\underline{\mathcal{M}} t}-P^{-} e^{\overline{\mathcal{M}} t}\right] R^{-} \leq \phi_{B}(t) P R^{-}} \\
& \leq\left[P^{+} e^{\overline{\mathcal{M}} t}-P^{-} e^{\underline{\mathcal{M}}} t\right] R^{-},
\end{aligned}
$$


since $R^{+} \geq 0$ and $R^{-} \geq 0$. Therefore, subtracting the previous inequalities and recalling that $R^{+}-R^{-}=R=$ $P^{-1}$, our choices $(5.21)$ of $\mathcal{L}_{a}(t)$ and $\mathcal{L}_{b}(t)$ give

$$
\begin{aligned}
\mathcal{L}_{a}(t) R^{+}-\mathcal{L}_{b}(t) R^{-} & \leq \phi_{B}(t) \\
& \leq \mathcal{L}_{b}(t) R^{+}-\mathcal{L}_{a}(t) R^{-} .
\end{aligned}
$$

For a suitable matrix that we denote by $*$, we have

$$
\phi_{B}(t)=\left[\begin{array}{cc}
\phi_{B_{1}}(t) & * \\
0 & \phi_{B_{3}}(t)
\end{array}\right] .
$$

Hence, (5.22) follows by left multiplying (5.28) through by $K$, and right multiplying (5.28) through by $K^{\top}$.

Proposition 5.1 includes the framer result from Lemma 5.2 as a special case. To see why, define $\mathcal{H}$ as in (5.19), under the assumptions of Lemma 5.2. Then,

$$
0 \leq \underbrace{\left[\begin{array}{cc}
\frac{\mathcal{K}}{0} & 0 \\
0 & \underline{\mathcal{K}}
\end{array}\right]}_{\underline{\mathcal{M}}} \leq \mathcal{H}(t) \leq \underbrace{\left[\begin{array}{cc}
\overline{\mathcal{K}} & \overline{\mathcal{L}} \\
\overline{\mathcal{L}} & \overline{\mathcal{K}}
\end{array}\right]}_{\overline{\mathcal{M}}},
$$

so we apply Proposition 5.1 with $\mathcal{M}=\mathcal{H}$. Note that

$$
P=\left[\begin{array}{cc}
I_{n} & 0 \\
I_{n} & I_{n}
\end{array}\right]
$$

is such that

$$
\begin{aligned}
& P \mathcal{H}(t) P^{-1}=\left[\begin{array}{cc}
I_{n} & 0 \\
I_{n} & I_{n}
\end{array}\right]\left[\begin{array}{cc}
\mathcal{K}(t) & \mathcal{L}(t) \\
\mathcal{L}(t) & \mathcal{K}(t)
\end{array}\right]\left[\begin{array}{cc}
I_{n} & 0 \\
-I_{n} & I_{n}
\end{array}\right] \\
& =\left[\begin{array}{cc}
\mathcal{K}(t)-\mathcal{L}(t) & \mathcal{L}(t) \\
0 & \mathcal{K}(t)+\mathcal{L}(t)
\end{array}\right]
\end{aligned}
$$

Then we choose $B_{1}(t)=\mathcal{K}(t)-\mathcal{L}(t)$, and (5.22) reads

$$
e^{\underline{\mathcal{K}} t}-\frac{e^{(\overline{\mathcal{K}}+\overline{\mathcal{L}}) t}-e^{(\overline{\mathcal{K}}-\overline{\mathcal{L}}) t}}{2} \leq \phi_{B_{1}}(t) \leq \frac{e^{(\overline{\mathcal{K}}+\overline{\mathcal{L}}) t}+e^{(\overline{\mathcal{K}}-\overline{\mathcal{L}}) t}}{2}
$$

(by [16, Lemma A.1]), which agrees with the conclusion of Lemma 5.2. Therefore, Proposition 5.1 includes Lemma 5.2 as a special case. Proposition 5.1 also provides framers that are based on embedding an $n$ dimensional system into a larger system of dimension strictly less than $2 n$, and which are therefore beyond the scope of [16]. Here is an example where this occurs:

EXAmple 1. Consider the case of the oscillator

$$
E(t)=\omega(t)\left[\begin{array}{cc}
0 & 1 \\
-1 & 0
\end{array}\right]
$$

where $\omega(t):[0, \infty) \rightarrow[0, \infty)$ is a continuous function. We choose the everywhere Metzler matrix

$$
\mathcal{M}(t)=\omega(t)\left[\begin{array}{lll}
0 & 1 & 0 \\
0 & 1 & 1 \\
2 & 0 & 1
\end{array}\right]
$$

The eigenvalues of the matrix in (5.33) are $2, i$, and $-i$. Therefore, there is constant matrix $P \in \mathbb{R}^{3 \times 3}$ such that

$$
P \mathcal{M}(t) P^{-1}=\omega(t)\left[\begin{array}{ccc}
0 & 1 & 0 \\
-1 & 0 & 0 \\
0 & 0 & 2
\end{array}\right]
$$

This gives estimates for $\phi_{E}$ by embedding the dynamics in dimension 3, whereas [16] only implies that one gets an estimate for $\phi_{E}$ using a system in dimension 4.

The preceding approach for the oscillator can be generalized to many other systems that can be transformed into the triangular form from Proposition 5.1, using similarity transformations. To illustrate the basic ideas of how this can be done, we first consider the constant matrix case

$$
\dot{x}=A x
$$

for any matrix $A \in \mathbb{R}^{n \times n}$. However, we can replace $A$ by a bounded function $A(t)$ which may be uncertain, if we allow the constants $\zeta$ and $\lambda$ that follow to depend only on suitable bounds on $A(t)$ (analogously to Lemma 5.2). We let $V=(1 \ldots . .1)^{\top} \in \mathbb{R}^{n}$ be the column matrix whose entries are all 1's (so $V V^{\top}$ is a matrix of all 1 's), and we denote the rows of $A$ by $A_{i}$ for $i=1,2, \ldots, n$, so the system can be written as $\dot{x}_{i}=A_{i} x$ for $i=1,2, \ldots, n$. Let $\zeta$ and $\lambda$ be positive constants to be selected. Then

$$
\dot{x}_{i}=\left(A_{i}+\zeta V^{\top}\right) x-\zeta V^{\top} x \text { for } 1 \leq i \leq n .
$$

We can select $\zeta$ so that each entry of each row $A_{i}+\zeta V^{\top}$ is nonnegative. Hence, $A+\zeta V V^{\top}$ is Metzler. Also,

$$
-V^{\top} \dot{x}=-\lambda V^{\top} x+\left(\lambda V^{\top}-V^{\top} A\right) x
$$

Choosing $\lambda$ large enough, it follows that all the entries of the vector $\lambda V^{\top}-V^{\top} A$ are nonnegative.

Next, consider the system

$$
\left\{\begin{aligned}
\dot{y}_{i} & =\left(A_{i}+\zeta V^{\top}\right) y+\zeta z, 1 \leq i \leq n \\
\dot{z} & =\lambda z+\left(\lambda V^{\top}-V^{\top} A\right) y
\end{aligned}\right.
$$

with state space $\mathbb{R}^{n+1}$. This system can be written in the form $\dot{Z}=\mathcal{M} Z$ for a constant Metzler matrix $\mathcal{M}$, and we deduce from (5.36) and (5.37) that any solution of (5.35) is such that $y_{i}=x_{i}$ and $z=-V^{\top} x$ provide a solution of (5.38). Then we can apply our approach from [16] to designing framers for cooperative systems.

Next, consider a bounded function $A(t)$ valued in $\mathbb{R}^{n \times n}$. A time-varying analog of the preceding approach provides positive constants $\zeta$ and $\lambda$ such that

$$
\mathcal{M}(t)=\left[\begin{array}{cc}
A(t)+\zeta V V^{\top} & \zeta V \\
\lambda V^{\top}-V^{\top} A(t) & \lambda
\end{array}\right]
$$


valued in $\mathbb{R}^{(n+1) \times(n+1)}$ is Metzler for all $t \geq 0$. Let

$$
P=\left[\begin{array}{cc}
I_{n} & 0 \\
V^{\top} & 1
\end{array}\right] \in \mathbb{R}^{(n+1) \times(n+1)}
$$

We then define $\mu$ by

$$
\mu(t)=P \mathcal{M}(t) P^{-1}=\left[\begin{array}{cc}
A(t) & \zeta V \\
0 & \lambda+n \zeta
\end{array}\right]
$$

Thus, we have an $n+1$ dimensional upper triangular form that is covered by Proposition 5.1, so we get framers for the solutions of $\dot{x}=A(t) x$ using solutions of $\dot{Y}=\mu(t) Y$. In particular, we only need to add a onedimensional dynamic extension. In the next section, we use our framers to design continuous-discrete observers.

\section{Framers and Continuous-Discrete Observers}

We next discuss our new solution in [16] to the problem of constructing exponentially stable continuous-discrete observers. Let $\underline{\nu}>0$ and $\bar{\nu}>\underline{\nu}$ be any two constants, and fix any sequences $\left\{t_{i}\right\}$ and $\left\{\nu_{i}\right\}$ in $[0, \infty)$ such that

$$
\begin{aligned}
& t_{0}=0, \text { and } t_{i+1}=t_{i}+\nu_{i} \text { and } \\
& \nu_{i} \in[\underline{\nu}, \bar{\nu}] \text { for all } i \in \mathbb{N} .
\end{aligned}
$$

The $t_{i}$ 's will serve as the measurement times for

$$
\left\{\begin{array}{l}
\dot{x}_{*}(t)=A_{*} x_{*}(t)+\varphi_{*}\left(t, x_{*}(t)\right) \\
y_{*}(t)=C_{*} x_{*}\left(t_{k}\right), t \in\left[t_{k}, t_{k+1}\right), k \in \mathbb{N}
\end{array}\right.
$$

with discrete measurements, where $x_{*}$ and $y_{*}$ are valued in $\mathbb{R}^{n}$ and $\mathbb{R}^{p}$ respectively. Assume:

Assumption 4. There is an invertible matrix $P \in$ $\mathbb{R}^{n \times n}$ such that the matrix $A=P A_{*} P^{-1}$ is Metzler. Also, $\varphi_{*}$ is $C^{1}$, and $\left(\partial \varphi_{*} / \partial x\right)(t, x)$ is bounded.

$$
\text { Set } \varphi(t, x)=P \varphi_{*}\left(t, P^{-1} x\right), C=C_{*} P^{-1} \text {, and }
$$

$$
w(t, a, b)=\int_{0}^{1} \frac{\partial \varphi}{\partial x}(t, r(b-a)+a) \mathrm{d} r .
$$

Then the Fundamental Theorem of Calculus gives $\varphi(t, b)-\varphi(t, a)=w(t, a, b)(b-a)$ for all $t \geq 0, a \in \mathbb{R}^{n}$, and $b \in \mathbb{R}^{n}$. Also, Assumption 4 provides positive constants $\bar{v}_{i j}$ such that each entry of $w=\left[w_{i j}\right]$ satisfies $w_{i j}(t, a, b) \in\left[-\bar{v}_{i j}, \bar{v}_{i j}\right]$ for all $t \geq 0, a \in \mathbb{R}^{n}$, $b \in \mathbb{R}^{n}, i \in\{1, \ldots, n\}$, and $j \in\{1, \ldots, n\}$. Set $\bar{D}=$ $\operatorname{diag}\left\{\bar{v}_{11}, \ldots, \bar{v}_{n n}\right\} \in \mathbb{R}^{n \times n}, \bar{V}=\left[\bar{v}_{i j}\right] \in \mathbb{R}^{n \times n}$, and

$$
\begin{aligned}
& \underline{\beta}(\rho)=e^{(A-\bar{D}) \rho}+\frac{1}{2}\left[e^{(A+\bar{D}) \rho}-e^{(A+2 \bar{V}-\bar{D}) \rho}\right] \\
& \text { and } \bar{\beta}(\rho)=\frac{1}{2}\left[e^{(A+2 \bar{V}-\bar{D}) \rho}+e^{(A+\bar{D}) \rho}\right]
\end{aligned}
$$

Using our bounds $\underline{\nu}$ and $\bar{\nu}$ from (6.42), we also assume:
Assumption 5. There exist a constant matrix $K \in$ $\mathbb{R}^{n \times p}$, a constant $\kappa \in(0,1)$, and a symmetric positive definite matrix $Q \in \mathbb{R}^{n \times n}$ such that for each constant matrix $\beta \in \mathbb{R}^{n \times n}$ satisfying

$$
\underline{\beta}(\rho) \leq \beta \leq \bar{\beta}(\rho) \text { for all } \rho \in[\underline{\nu}, \bar{\nu}],
$$

the inequality $\beta^{\top}(I-K C)^{\top} Q(I-K C) \beta \preceq \kappa Q$ holds.

See Section 7 for ways to verify Assumption 5. In [16], we prove the following, where $\hat{x}\left(t_{0}^{-}\right)=\hat{x}\left(t_{0}\right)$ :

THEOREM 6.1. Let the system (6.43) satisfy Assumptions 4-5 and choose the continuous-discrete system

$$
\left\{\begin{aligned}
\dot{\hat{x}}_{*}(t)= & A_{*} \hat{x}_{*}(t)+\varphi_{*}\left(t, \hat{x}_{*}(t)\right) \\
& \text { for all } t \in\left[t_{k}, t_{k+1}\right) \\
\hat{x}_{*}\left(t_{k}\right)= & \hat{x}_{*}\left(t_{k}^{-}\right)+P^{-1} K\left[y_{*}\left(t_{k}\right)-P \hat{x}_{*}\left(t_{k}^{-}\right)\right]
\end{aligned}\right.
$$

for all $k \geq 0$. Then the dynamics for the observation error $x_{*}-\hat{x}_{*}$ is uniformly globally exponentially stable to 0 .

REMARK 1. We can always rewrite the $x_{*}$ dynamics in (6.43) as $\dot{x}_{*}(t)=\Psi_{*}\left(t, x_{*}(t)\right)$, where $\Psi_{*}\left(t, x_{*}\right)=$ $A_{*} x_{*}+\varphi_{*}\left(t, x_{*}\right)$. Hence, we can replace Assumption 4 by the requirements that $\varphi_{*}$ is $C^{1}$ and $\left(\partial \varphi_{*} / \partial x\right)(t, x)$ is bounded and select $A_{*}=0$. However, different $A_{*}$ 's and $P$ 's produce different conditions in Assumption 5, so it is helpful to consider different possible $A_{*}$ 's. The proof of Theorem 6.1 provides explicit formulas for the functions in the final exponential stability estimate.

REMARK 2. The functions (6.45) are obtained by setting $\underline{\mathcal{K}}=A-\bar{D}, \overline{\mathcal{K}}=A+\bar{V}$, and $\overline{\mathcal{L}}=\bar{V}-\bar{D}$ in (5.17). They correspond to choosing $\mathcal{M}(t)=\mathcal{K}(t)-$ $\mathcal{L}(t)=A+V(t)$ in Lemma 5.2 for each $i$, where $\mathcal{K}(t)=A_{V}(t), \mathcal{L}(t)=V_{q}(t), A_{V}(t)=A+D_{V}(t)+V_{p}(t)$, $D_{V}(t)=\operatorname{diag}\left\{v_{11}, v_{22}, \ldots, v_{n n}\right\}, V(t)=\left[v_{i j}(t)\right]=$ $w\left(t, P x_{*}(t), P \hat{x}_{*}(t)\right)$ for any fixed solutions of the system and observer, $V_{p}(t)=\max \left\{V_{N}(t), 0\right\}, V_{q}(t)=$ $\max \left\{V_{N}(t), 0\right\}-V_{N}(t)$, and $V_{N}(t)=V(t)-D_{V}(t)$. They provide framers for the dynamics for the error variable $\bar{x}=P\left(\hat{x}_{*}-x_{*}\right)$ on $\left[t_{i}, t_{i+1}\right)$ for all $i \geq 0$. However, they can be replaced by our new framers from Proposition 5.1, using arguments from the preceding section. This produces different versions of Theorem 6.1 for different framers, which were not considered in [16].

\section{LMI Formalism}

In applications, it can be convenient to check Assumption 5 using LMIs. To see why, we define the functions $\bar{\beta}$ and $\beta$ by $(6.45)$, and we introduce the sets of matrices

$$
\begin{aligned}
& \mathcal{F}(\rho)= \\
& \left\{\beta \in \mathbb{R}^{n \times n}: \beta_{i j} \in\left\{\underline{\beta}_{i j}(\rho), \bar{\beta}_{i j[}(\rho)\right\} \text { for all } i \text { and } j\right\} .
\end{aligned}
$$


Following [9], this allows us to rewrite Assumption 5 as an LMI, as in the following result from [16]:

Proposition 7.1. Assume that there exist a symmetric positive definite matrix $Q \in \mathbb{R}^{n \times n}$ and a matrix $W \in \mathbb{R}^{n \times p}$ such that

$$
\left[\begin{array}{cc}
Q & (Q-W C) \beta \\
\beta^{\top}(Q-W C)^{\top} & Q
\end{array}\right] \succ 0
$$

holds for all $\beta \in \mathcal{F}(\rho)$ and all $\rho$ in $[\underline{\nu}, \bar{\nu}]$. Then there is a constant $\kappa \in(0,1)$ such that Assumption 5 holds with $K=Q^{-1} W$.

Moreover, if a condition ensuring the existence of an observer in the case of continuous time measurement holds, then Assumption 5 holds provided the $\nu_{k}$ 's are small enough. This is made precise in the following proposition in [16]:

Proposition 7.2. Let Assumption 4 hold, and define $\bar{V}$ as in Section 6. Assume that there exist a matrix $K_{0} \in \mathbb{R}^{n \times p}$, a constant $\kappa_{0} \in(0,1)$, and a symmetric positive definite matrix $Q \in \mathbb{R}^{n \times n}$ such that

$$
\left(\beta_{0}-K_{0} C\right)^{\top} Q+Q\left(\beta_{0}-K_{0} C\right) \preceq-\kappa_{0} Q
$$

holds for all matrices $\beta_{0} \in \mathbb{R}^{n \times n}$ such that $A-\bar{V} \leq$ $\beta_{0} \leq A+\bar{V}$. Then there exists a constant $\nu_{*}>0$ such that for all $\nu \in\left(0, \nu_{*}\right]$, Assumption 5 is satisfied with the choices $\underline{\nu}=\bar{\nu}=\nu$ and $K=\nu K_{0}$.

\section{Conclusions and Open Problems}

This tutorial discussed several recent results on continuous-discrete observers. Such observers are useful for finding asymptotic estimators of the states of continuous time systems in situations where only discrete observations of an output of the system are available for measurement. While many results on observers have appeared in the literature, the authors' approaches are distinguished through their use of differential inclusions coupled with the fact that they provide framers for the original systems, i.e., explicit upper and lower bounds for the unknown states (in the vector sense) that hold for all times. The explicit framers in [16] are found by embedding $n$ dimensional systems as subsystems of larger $2 n$ dimensional systems.

This tutorial also included new original results on framers, which have not appeared before and which are not being submitted simultaneously for publication elsewhere. Our new framers include the framers in [16] as a special case, but are more general because they make it possible to obtain frames by embedding $n$ dimensional systems as subsystems of larger systems having dimension $n+1$. One research direction worth pursuing is to compare the performances of observers that are obtained using the framers from our new Proposition 5.1 with the observers that are obtained from the framers in the previously reported Lemma 5.2. Having sample output values can be viewed as having delayed output measurements, with a timevarying delay. It would be interesting to generalize our approaches to allow delays in the original plant, or cases where the original dynamics is a PDE.

\section{References}

[1] T. Ahmed-Ali, V. Van Assche, J. Massieu, and P. Dorleans. Continuous-discrete observer for state affine systems with sampled and delayed measurements. IEEE Transactions on Automatic Control, 58(4):1085-1091, 2013.

[2] T. Ahmed-Ali, R. Postoyan, and F. LamnabhiLagarrigue. Continuous-discrete adaptive observers for state affine systems. Automatica, 45(12):29862990, 2009.

[3] V. Andrieu. Extended-Kalman-filter-like observers for continuous time systems with discrete time measurements. http://hal.archives-ouvertes.fr/hal00461762.

[4] V. Andrieu and M. Nadri. Observer design for Lipschitz systems with discrete-time measurements. In Proc. IEEE Conference on Decision and Control, pages 6522-6527, Atlanta, GA, 2010.

[5] M. Arcak and D. Nesic. A framework for nonlinear sampled-data observer design via approximate discrete-time models and emulation. Automatica, 40(11):1931-1938, 2004.

[6] C.-M. Astorga, N. Othman, S. Othman, H. Hammouri, and T.-F. McKenna. Nonlinear continuousdiscrete observers: Applications to emulsion polymerization reactors. Control Engineering Practice, 10(1):3-13, 2002.

[7] F. Cacace, A. Germani, C. Manes, and R. Setola. A new approach to the internally positive representation of linear MIMO systems. IEEE Transactions on Automatic Control, 57(1):119-134, 2012.

[8] D. Deza, E. Busvelle, J.-P. Gauthier, and D. Rakotopara. High gain estimation for nonlinear systems. Systems and Control Letters, 18(4):295-299, 1992.

[9] T. Dinh, V. Andrieu, M. Nadri, and U. Serrres. Continuous-discrete time observer design for Lipschitz systems with sampled measurements. IEEE Transactions on Automatic Control, 60(3):787792, 2015.

[10] M. Farza, M. M'Saad, M. Fall, E. Pigeon, O. Gehan, and R. Mosrati. Continuous-discrete time observers for a class of MIMO nonlinear systems. In Proc. European Control Conf, pages 2146- 
2151, Zurich, Switzerland, 2013.

[11] J.-P. Gauthier, H. Hammouri, and S. Othman. A simple observer for nonlinear systems applications to bioreactors. IEEE Transactions on Automatic Control, 37(6):875-880, 1992.

[12] W. Haddad, V. Chellaboina, and Q. Hui. Nonnegative and Compartmental Dynamical Systems. Princeton University Press, Princeton, NJ, 2010.

[13] H. Hammouri, N. Nadri, and R. Mota. Constant gain observer for continuous-discrete time uniformly observable systems. In Proc. IEEE Conference on Decision and Control, pages 5406-5411, San Diego, CA, 2006.

[14] A. Jazwinski. Stochastic Processes and Filtering Theory. Courier Dover Publications, New York, 2007.

[15] I. Karafyllis and C. Kravaris. From continuous-time design to sampled-data design of observers. IEEE Transactions on Automatic Control, 54(9):21692174, 2009.

[16] F. Mazenc, V. Andrieu, and M. Malisoff. Design of continuous-discrete observers for time-varying nonlinear systems. Automatica, accepted.
[17] F. Mazenc and T. Dinh. Construction of interval observers for continuous-time systems with discrete measurements. Automatica, 50(10):25552560, 2014.

[18] M. Nadri and H. Hammouri. Design of a continuous-discrete observer for state affine systems. Applied Mathematics Letters, 16(6):967-974, 2003.

[19] A. Tellez-Anguiano, C. Astorga-Zaragoza, E. Alcorta-Garcia, B. Targui, E. Quintero-Marmol, M. Adam-Medina, and V. Olivares-Peregrino. Nonlinear continuous-discrete observer application to distillation columns. International Journal of Innovative Computing, Information and Control, 8(1B):763-778, 2012.

[20] A. Zemouche and M. Boutayeb. On LMI conditions to design observers for Lipschitz nonlinear systems. Automatica, 49(2):585-591, 2013.

[21] A. Zemouche, M. Boutayeb, and G. Bara. Observers for a class of Lipschitz systems with extension to $H_{\infty}$ performance analysis. Systems and Control Letters, 57(1):18-27, 2008. 